Rodolfo José Gomes de Araújo ${ }^{1}$, Layla Cristine Gomes de Oliveira ${ }^{2}$, Leila Maués Oliveira Hanna ${ }^{3}$, Adriano Maia Corrêa ${ }^{4}$, Liliane Helena Vilar Carvalho ${ }^{5}$, Nair Carolina Ferreira Alvares ${ }^{6}$

1. Mestre, Professor de Periodontia da Universidade Federal do Pará - UFPA Belém (PA), Brasil.

2. Pós-graduanda em Biologia dos Agentes Infecciosos e Parasitários da Universidade Federal do Pará e Professora de Periodontia da Universidade Federal do Pará - UFPA - Belém (PA), Brasil. 3. Pós-graduanda em Odontologia da Universidade Federal do Pará - UFPA -Belém (PA), Brasil.

4. Doutor, Professor de Periodontia da Universidade Federal do Pará - UFPA Belém (PA), Brasil.

5. Acadêmica do Curso de Graduação em Odontologia do Centro Universitário do Pará - CESUPA - Belém (PA), Brasil. 6. Acadêmica do Curso de Graduaçáo em Odontologia da Universidade Federal do Pará - UFPA - Belém (PA), Brasil.

Recebido da Universidade Federal do Pará - UFPA - Belém (PA), Brasil. Trabalho apresentado na 25a Reunião Anual da Sociedade Brasileira de Pesquisa Odontológica, 2008, Águas de Lindóia. [ Gomes de Araújo RJ, Gomes de Oliveira LC Hanna LMO, Corrêa AM. Análise de ações e percepçóes sobre cuidados bucais realizados por equipes de enfermagem em unidades de tratamento intensivo. In: $25^{\text {a }}$ Reunião Anual da Sociedade Brasileira de Pesquisa Odontológica, 2008, Águas de Lindóia. Brazilian Oral Research. São Paulo, 2008. v. 22. p. 27-27].

Submetido em 26 de Junho de 2008 Aceito em 21 de Fevereiro de 2009

Autor para correspondência: Rodolfo José Gomes de Araújo Tv. Rui Barbosa 1533 / 302 - Nazaré CEP: 66035-220 - Belém (PA), Brasil. Fone: (91) 3225-3130 / (91) 8142-0444 E-mail: rodolfogomesaraujo@uol.com.br

\section{Análise de percepçóes e açóes de cuidados bucais realizados por equipes de enfermagem em unidades de tratamento intensivo}

\author{
Perceptions and actions of oral care performed by nursing teams \\ in intensive care units
}

\section{RESUMO}

Objetivos: Buscando estabelecer um perfil da percepçáo e realizaçáo dos cuidados em saúde bucal prestados a pacientes internados em unidades de tratamento intensivo por equipes de enfermagem, realizou-se um estudo com entrevistas orientadas por um questionário.

Métodos: A população de estudo foi constituída de profissionais de enfermagem divididos em três categorias de formação: enfermeiros, técnicos de enfermagem e auxiliares de enfermagem que atuam em instituiçóes hospitalares públicas e particulares prestando serviços em unidades de tratamento intensivo na cidade de Belém - PA.

Resultados: A pesquisa desenvolvida neste trabalho retornou com resultados, os quais sugerem que os cuidados de higiene bucal realizados nos pacientes hospitaliza- dos em unidades de terapia intensiva são escassos e inadequados, sendo necessárias modificaçôes nos cuidados dispensados atualmente, especialmente no ambiente nosocômial da equipe de atençáo ao paciente.

Conclusão: A presença de cirurgiáo dentista, a difusão dos conhecimentos de específicos de higiene bucal são medidas sugeridas como tentativas de solucionar as dificuldades apresentadas na manutenção da saúde bucal e no tratamento das doenças bucais, que afetam a saúde geral dos pacientes hospitalizados. A atuação interdisciplinar no atendimento a estes indivíduos é defendida visando à obtenção de uma melhor qualidade de vida prevenindo ou minimizando patologias orais presentes.

Descritores: Equipe de assistência ao paciente; Higiene bucal/enfermagem; Unidades de terapia intensiva odontologia preventiva e o uso de recursos

\section{INTRODUÇÁO}

As unidades de terapia intensiva (UTIs) foram criadas a partir da necessidade de atendimento do cliente cujo estado crítico exigia assistência e observação contínua de médicos e enfermeiros e da necessidade de aperfeiçoamento e concentração de recursos materiais e humanos para o atendimento a pacientes graves, mas tidos ainda como recuperáveis, e da necessidade de observação constante, centralizando os pacientes em um núcleo especializado. ${ }^{(1)}$

Equipes interdisciplinares (profissionais de áreas afins entre si) e multidisciplinares (em conjunto com profissionais de áreas especializadas fazem parte do dia a dia destas unidades.

O paciente internado na UTI necessita de cuidados de excelência, dirigidos não apenas para os problemas fisiopatológicos, mas também para as questóes psicossociais, ambientais e familiares que se tornam intimamente interligadas à doença física. A essência da multidisciplinaridade, profissionais de diferentes áreas atuando sobre um mesmo paciente, em cuidados intensivos não está nos ambientes 
ou nos equipamentos especiais, mas no processo de tomada de decisōes, baseado na sólida compreensão das condiçóes fisiológicas, psicológicas do pacientes e novas terapias.

É essencial que pacientes de UTIs tenham cuidados de higiene oral suficientes durante sua internação com o objetivo de prevenir a instalação de patologias orais e possíveis complicações de doenças bucais já existentes. ${ }^{(2)}$

Em pacientes hospitalizados, patógenos comumente responsáveis pela pneumonia nosocomial sáo encontrados colonizando placa dental e mucosa bucal destes pacientes. Porém, boas técnicas de higiene bucal são capazes de prevenir o avanço da infecção da cavidade bucal para o trato respiratório. ${ }^{(3)}$

Em aproximadamente 24 horas sem limpeza da cavidade oral é possível detectar clinicamente uma camada de placa dental. E a ausência ou a técnica de higiene bucal adotada será intimamente ligada ao número e à espécie de microorganismo encontrado na cavidade oral. ${ }^{(4)}$

Observou-se a rota de colonização traqueal no desenvolvimento da pneumonia associada à ventilaçáo e constatou que 80 de 100 pacientes tiveram colonizaçáo durante o primeiro dia de ventilação endotraqueal. $\mathrm{E}$ a cavidade oral é a primeira fonte de organismos patogênicos que causam esta patologia. ${ }^{(5)}$

Vários estudos têm documentado que indivíduos hospitalizados tendem a apresentar higiene bucal deficiente, em comparação com os pacientes ambulatoriais e os pacientes controles da sociedade. Essa ausência de atenção com a higiene bucal resulta no aumento da quantidade e complexidade da placa dental, que pode favorecer a interação bacteriana entre bactérias indígenas da placa e patógenos respiratórios conhecidos, como Pseudomonas aeruginosa e bacilos entéricos. ${ }^{(6)}$

Foram realizados estudos que avalia a prevalência de patógenos respiratórios colonizando a boca em um grupo de pacientes internados em UTI, com uma atençáo especial à placa dental e à mucosa. Os autores observaram que $65 \%$ dos pacientes examinados, a placa e a mucosa bucal apresentavam patógenos respiratórios, entre eles Staphylococcus aureus, Pseudomonas aeruginosa e 10 gêneros de bacilos gram-negativos. Esses pacientes apresentavam higiene oral deficiente, o que pode, segundo o autor, influenciar na colonizaçáo da orofaringe por microorganismos respiratórios e também há a hipótese de que a flora normal da boca é alterada devido à algumas enzimas que presentes na superfície da mucosa, deixando-a mais favorável à aderência desses patógenos. ${ }^{(7)}$

Foram analisados os cuidados com a orofaringe $16 \mathrm{pa}$ cientes internados em UTIs que faziam uso de ventilação mecânica. Dados clínicos e demográficos eram registrados a cada dia de internação através de coletas de microorganis- mos com swabs, secreção traqueal e exames clínicos orais até os aparelhos de ventilação fossem retirados. Sete pacientes apresentaram xerostomia; dez lesóes de lábios foram identificadas em nove pacientes; oito lesôes de língua foram diagnosticadas em nove pacientes; e oito lesóes de mucosa foram registradas em nove pacientes. Quarenta e quatro por cento dos pacientes demonstraram radiograficamente evidencias consistentes com pneumonia. Microorganismos gram negativos foram evidenciados na orofaringe e na secreção traqueal simultaneamente. Embora a higiene da cavidade oral seja considerada um procedimento com alto grau de dificuldade para ser realizado em pacientes entubados, sabe-se que o mal estado da orofaringe pode ser relacionado com a aquisição de organismos nosocomiais e a esta relação deveria ser dada maior atenção. Portanto a análise sistemática e clinica da orofaringe pode prevenir o surgimento e agravamento de muitas infecções. ${ }^{(8)}$

O tratamento da orofaringe e a manutenção de uma higiene favorável sáo procedimentos difíceis de serem realizados em pacientes sob cuidados intensivos, principalmente naqueles que fazem uso de ventilação mecânica devido ao difícil acesso na cavidade oral..$^{(9)} \mathrm{O}$ paciente intubado possui maiores riscos de serem vitimas de colonizaçóes de microorganismos, pois a cavidade oral está em contato com outros instrumentais como: fitas, afastadores bucais, tubos entre outros. ${ }^{(8)} \mathrm{A}$ função do tubo oral endotraqueal é propiciar ventilaçáo e proteção para passagem de ar. A posiçấo do tubo e de outros materiais de suporte pode obstruir a visualização da cavidade oral e limitar o acesso influenciando negativamente no processo de higiene. ${ }^{(10)}$ Desta forma, profissionais de enfermagem se sentem relutantes a manipular o aparato necessário a respiração do pacientes para realizar procedimentos de higiene. Por sua vez, as fitas de fixação presentes muito próximas da cavidade oral rapidamente se tornam fortemente contaminadas por patógenos quando moléculas de saliva são manipuladas na tentativa de realizaçấo de técnicas de higiene. ${ }^{(11)}$

Apesar da existência de importantes recursos como saliva artificial, sugadores, antissépticos, escovas dentais elétricas e raspadores de língua que podem ser utilizados para tratamento oral em UTIs, estes raramente são usados. Dentre as razões discutidas pela não utilização de tais ferramentas, a falta de tempo disponível ou conhecimento do profissional responsável, e a falta de assistência de profissionais especializados em saúde bucal dentro da UTI, são as mais relatadas. ${ }^{(12)}$ Interaçóes com técnicos de higiene dental poderiam aprimorar o conhecimento e habilidade da equipe de enfermagem em relação a cuidados orais. ${ }^{(1)}$

Higiene oral em UTIs é considerada um procedimento básico, indispensável de enfermagem cujo objetivo é manter a cavidade do pacientes saudável. Tais procedimentos são 
necessários para: obter e manter limpeza; prevenir infecçōes / estomatites; manter a mucosa oral úmida; promover conforto ao paciente. ${ }^{(13,14)}$

Em estudos com enfermeiras, diretores de hospitais e enfermeiras domiciliares, constataram vários conceitos incorretos sobre práticas de cuidados dentais em pacientes hospitalizados. A falta de conhecimento sobre patologias odontológicas foi considerada ampla, abrangendo inúmeros aspectos como o exame da cavidade oral. As dificuldades de locomoção e outras limitaçóes físicas dos pacientes são encaradas como as maiores dificuldades ou impossibilidades para o cuidado odontológico. Foi estimado que $48 \%$ dos profissionais que participaram da pesquisa não tinham tido acesso a esses conhecimentos e $30 \%$ da amostra demonstrou que estes não eram assuntos prioritários em suas funçóes. ${ }^{(15)}$ Foi verificado que $83 \%$ do corpo de enfermagem não recebeu treinamentos básicos acerca de saúde oral. Os pesquisadores atentam para a necessidade de melhoria da qualidade dos cuidados orais de pacientes geriátricos e concluem que treinamentos apropriados e a presença do dentista no ambiente hospitalar contribuem para o aperfeiçoamento dos conhecimentos e para melhor desempenho da enfermagem em relação à saúde bucal dos pacientes. ${ }^{(16)}$

O presente trabalho teve como objetivo avaliar as percepçóes e açóes da equipe de enfermagem, quanto aos cuidados de saúde bucal prestados aos pacientes internados em unidades de tratamento intensivo durante o processo de higienização bucal diário ao qual têm sido submetidos.

\section{MÉTODOS}

Visando à obtenção de um perfil da percepção dos cuidados bucais por equipes de enfermagem, fez-se uma pesquisa com abordagem estatística de análise de dados, envolvendo como população alvo profissionais de enfermagem que atuavam em UTIs de hospitais públicos e particulares localizados na cidade de Belém - PA, durante o período de junho a novembro de 2007. É lícito esclarecer que foram consideradas instituiçóes destinadas a pacientes adultos com o mínimo de oito leitos disponíveis para utilização.

Optou-se por não dividirem grupos distintos já que a comparação entre hospitais públicos e particulares não caracterizava objetivo do trabalho e a divisão entre grupos comprometeria a exatidão e a coerência dos resultados.

Após submissão e registro da aprovação do comitê de ética em pesquisa em seres humanos do centro de ciências da saúde da Universidade Federal do Pará, a pesquisa foi, entáo, desenvolvida por meio de entrevista realizada por único entrevistador, cirurgião-dentista, utilizando questionário estruturado.
Foram entrevistados profissionais de enfermagem, de ambos os sexos, sem limites quanto idade, que atuavam em 23 unidades de tratamento intensivo pertencentes a 12 instituições públicas e privadas de saúde em Belém, capital do estado do Pará.

O questionário foi formulado com 18 questôes de múltipla escolha. Este número foi determinado procurandose fazer menção aos assuntos mais significativos uma vez que os profissionais estariam em seu horário de trabalho e não disponibilizariam muito tempo para responder ao documento em questáo. $\mathrm{O}$ tempo médio das entrevistas foi cronometrado previamente e registrou-se que aproximadamente quatro minutos e trinta segundos seriam necessários para a participação de cada membro escolhido.

A primeira parte do questionário, representada pelas questóes de 01 a 07 , teve como objetivo avaliar o perfil do corpo de enfermagem entrevistado quanto à categoria profissional, faixa etária, gênero, rendimentos, tipo de instituição em que trabalha e formação do profissional.

As questóes seguintes, de 8 a 11 , fizeram referência à participação em equipe multidisciplinar, ao contato ou não com um cirurgiâo dentista e ao nível de conhecimento da equipe de enfermagem no que diz respeito à odontologia $\mathrm{e}$ sua relaçáo com a saúde geral do indivíduo.

Foram avaliados, a partir da questáo 12 a 17, os conhecimentos e os cuidados que são realizados rotineiramente no ambiente das UTIs ou orientados aos pacientes durante a permanência no hospital. Finalmente, avaliamos com a questão 18 o nível de interesse pelo corpo de enfermagem em receber orientaçóes sobre saúde bucal e aplicá-las clinicamente.

Para a aplicação do questionário, o entrevistador visitou as instituiçôes hospitalares portando uma carta elaborada pelo orientador do projeto de pesquisa, propondo-se a demonstrar os objetivos do estudo. Após divulgação do parecer positivo da diretoria do hospital registrando a autorização para a realização das entrevistas, os questionários eram entregues individualmente aos membros da equipe de enfermagem. Aguardava-se que o profissional respondesse às questóes e as eventuais dúvidas eram solucionadas pelo entrevistador, assegurando respostas conscientes e seguras dos entrevistados. Importante mencionar que as visitas aos hospitais ocorreram em todos os turnos (manhã, tarde e noite), seguindo escalas de trabalho estabelecidas por cada instituição hospitalar. Retornos às instituiçóes eram necessários quando se notava que não estavam presentes todos os profissionais em atividade naquele período, objetivando-se abordar as equipes de enfermagem em sua totalidade. Profissionais que estavam em períodos de férias ou em uso de licenças profissionais não constaram em nossos relatórios como parte do público-alvo do estudo. Os entrevistados 
foram orientados e informados, por meio do termo de consentimento livre e esclarecido, quanto à não-obrigatoriedade de sua participação, assim como quanto à garantia de sigilo absoluto em relação a sua identidade e nome do local de trabalho em que atuavam.

\section{RESULTADOS}

Foram realizadas 402 entrevistas com membros da equipe de enfermagem, sendo 73 enfermeiros, 284 técnicos e 45 auxiliares de enfermagem. As respostas foram tabuladas e submetidas à análise estatística conforme descrevemos abaixo. Verificamos que, em todas as categorias, a maioria dos profissionais atua em equipes interdisciplinares conforme assinala a tabela 1 .

Com relação à presença de um cirurgiáo dentista efetivo na equipe interdisciplinar que quase a totalidade dos entrevistados respondeu que as equipes das quais faziam parte não dispunham de cirurgião-dentista. E, ainda, $86 \%$ dos entrevistados consideram necessária a presença deste profissional na equipe, que poderia atuar, assim, nos casos onde houvesse envolvimento odontológico.

No que diz respeito ao conhecimento da relação saúdebucal/saúde-geral, $99 \%$ dos entrevistados concordaram com a afirmativa de que uma infecçáo na boca pode fazer com que a saúde do resto do corpo seja prejudicada. Além disso, $99,2 \%$ dos entrevistados acreditam que a higienização da boca é importante durante a estada no hospital.

Podemos observar que a realizaçáo do exame da cavidade bucal e/ou orientação para procurar um profissional da odontologia para realizá-lo não é executada de acordo com as respostas fornecidas por $37 \%$ dos profissionais. Para um grupo, que representa um percentual de aproximadamente $63 \%$ dos entrevistados, o exame oral e/ou orientação é realizado.

Os profissionais entrevistados também responderam à questóes em relaçáo ao fato de realizarem e orientarem seus pacientes segundo os seguintes procedimentos: escovaçáo dental normal (sem auxílio de pessoas ou recursos especiais); uso de cuba para escovação no leito. Verificou-se que $58 \%$ dos entrevistados afirmaram realizar e/ou orientar seus pacientes em relação a escovação dental normal. Quando solicitado aos entrevistados, em questão aberta, que nomeassem a marca de colutórios mais utilizada, obtivemos o índice de 100,00\% de resposta para cloreto de cetilpiridínio.

Recursos sob a forma de gel, spray, creme dental, enxaguatório e até chicletes, que atuam sobre os tecidos bucais suavizando os efeitos da boca seca, são pouco divulgados na classe de enfermagem: aproximadamente $90 \%$ dos entrevistados relatam nunca terem ouvido falar desses produtos e, quanto ao uso em hospitais, nenhum deles narrou alguma experiência isolada de contato com esses recursos.

Avaliando o conhecimento dos profissionais de enfermagem a respeito de diversos temas odontológicos, aproximadamente $30 \%$ dos entrevistados responderam que têm conhecimento sobre técnicas de escovação dentária. Aproximadamente $76 \%$ afirmam reconhecer os aspectos normais da cavidade bucal e quando o assunto é as doenças mais comuns da cavidade bucal, como cárie dental, gengivite, periodontite e candidíase, 29\% desconhecem. Dentre os entrevistados, 30\% julgam saber sobre higienização das próteses e interrupção do uso destas e $29 \%$ afirmam ter conhecimentos sobre higiene das mucosas. Quanto à limpeza da língua, aproximadamente $40 \%$ dizem conhecer bem o assunto e afirmam sua capacidade em orientar os pacientes, caso sejam questionados.

Foi questionada a realização de treinamentos específicos sobre higiene bucal durante a formação profissional dos membros da equipe de enfermagem. Observamos que aproximadamente $42 \%$ dos profissionais receberam algum certo conhecimento a respeito do assunto.

Em todas as categorias a classificação quanto à eficácia do treinamento realizado é apontada como insuficiente por $74 \%$ da amostra. $\mathrm{O}$ interesse por conhecimentos sobre saúde bucal foi de $98 \%$.

\section{DISCUSSÁO}

No que diz respeito ao cuidado direto do paciente, a maioria dos estabelecimentos conta, em seu quadro de profissionais, com médicos em diversas especialidades, enfermeiros, técnicos e auxiliares de enfermagem. Outros profissionais são encontrados em menor freqüência, tais como fisioterapeutas,

Tabela 1 - Você atua em equipe multi/interdisciplinar?

\begin{tabular}{lcccccc}
\hline & \multicolumn{2}{c}{ Sim } & \multicolumn{2}{c}{ Náo } & \multicolumn{2}{c}{ Total } \\
& Frequência & $\mathbf{\%}$ & Frequência & $\mathbf{\%}$ & Frequência & \% \\
\hline Enfermeira & 72 & 17,9 & 1 & 0,2 & 73 & 18,2 \\
Técnico de enfermagem & 277 & 68,9 & 7 & 1,7 & 284 & 70,6 \\
Auxiliar de enfermagem & 45 & 11,2 & 0 & 0,0 & 45 & 11,2 \\
\hline Total & 394 & 98,0 & 8 & 2,0 & 402 & 100,0 \\
\hline
\end{tabular}


nutricionistas e psicólogos.

A partir do questionamento da presença de um cirurgiãodentista efetivo, que seria o responsável direto por casos relativos ao envolvimento odontológico consideramos, primeiramente, que os hospitais deveriam rever seus conceitos de equipe interdisciplinar, para que o ingresso de um cirurgiáodentista no ambiente hospitalar possa ser justificado. ${ }^{(1,2)}$

Podemos notar, por meio da análise estatística deste trabalho, que, mesmo náo tendo suas atençóes voltadas para a prevenção e cuidados de higiene bucal, quando ressalta que os cirurgiōes dentistas, de certa forma, com o conhecimento das enfermeiras. ${ }^{(17)} \mathrm{A}$ presença de um cirurgião-dentista nas equipes hospitalares é necessária para $86 \%$ dos entrevistados que reconhecem que o dentista poderia atuar nos casos onde houvesse envolvimento odontológico. A literatura sobre a odontologia na prática interdisciplinar, especificamente em UTIs, é recente no Brasil, havendo poucas mençóes de dentistas atuando nesse setor antes da década de 90 , em contradição com muitos outros países. Entretanto, em virtude do crescimento do número de pesquisas científicas no ramo da odontologia relacionando alteraçôes bucais com tantas outras alteraçóes orgânicas, ocorre um concomitante aumento de interesse pela chamada "odontologia hospitalar".

Durante as visitas que foram realizadas nas UTIs, observou-se que alguns pacientes podem não necessitar do uso de ventilação mecânica e estarem aptos a realizar seus próprios cuidados de higiene básica, requerendo ajuda por parte da enfermagem É importante avaliar, portanto, o grau de independência dos pacientes, pois o autocuidado deve ser estimulado e a independência do paciente encorajada, não com o intuito de se reduzir as obrigaçóes da enfermagem, mas de estimular a recuperação do paciente. Ressalta-se a importância da verificação do uso de próteses, as quais no momento da internação e identificação não estão sendo utilizadas. Nesses casos, recomenda-se que sejam mantidas em copos etiquetados. Os dados obtidos nesta pesquisa demonstraram que aproximadamente $100 \%$ dos pacientes náo utilizam suas próteses, ainda que não necessitem de ventilação mecânica. ${ }^{(18)}$ $\mathrm{Na}$ maioria dos casos verificou-se que as próteses eram entregues a família ou acompanhantes no momento da internaçáo nas emergências e UTIs devido aos procedimentos de emergência, contrariando pesquisadores que chamaram a atenção para a importância do uso destes utensílios sempre que possível no ambiente hospitalar para prevenção de uma variedade de alteraçôes que podem ser provocadas, como modificaçóes na mucosa, diminuição da dimensão vertical de oclusão e reabsorção de osso alveolar.

O paciente deverá ter uma higiene bucal adequada mesmo na ausência de dentes. Os autores sugerem massagens para fortalecer as gengivas e a lavagem das próteses para remoçáo dos detritos alimentares que podem ferir o paciente $\mathrm{e}$ incomodá-lo. Acrescenta-se, ainda, que seja de responsabilidade da enfermeira a observação do estado bucal do paciente, constatando se ele usa próteses, se pode alimentar-se sozinho e se tem capacidade mastigatória. ${ }^{(14)}$ Os dados obtidos nesta pesquisa auxiliarão a avaliação sobre a importância do profissional de odontologia no ambiente das UTIs. A formação de suas perguntas foi direcionada para o conhecimento das opinióes dos profissionais de enfermagem sobre sua capacidade em realizar procedimentos de higiene oral em seus pacientes da importância de um odontólogo que os orientasse ou realizasse tais práticas.

As orientaçóes sobre os cuidados bucais deverão ser adequadas às habilidades motoras e capacidade cognitiva do paciente. Desse modo, os propósitos preventivos devem ser compreendidos pelo paciente e por seu grupo de apoio (familiares e/ou enfermagem). Muitas vezes, causas físicas ou mentais impedem uma higiene bucal satisfatória, sendo necessário o auxílio de alguém devidamente treinado. Um guia que pode também ser sugerido à equipe de enfermagem sobre a relaçáo entre a necessidade de procedimentos adequados de higiene bucal e diferentes níveis de dependência dos pacientes encontra-se no quadro 1.

A prevenção e a promoção de saúde bucal em UTI é destacada por meio de protocolos elaborados de acordo com as necessidades individuais dos pacientes internados em UTIs, recomendando a remoção mecânica da placa nos indivíduos dentados utilizando, para esse fim, escovas dentais corretamente indicadas. Porém nenhuma das equipes observadas obedecia algum tipo de protocolo individual referente à hi-

Quadro 1 - Procedimentos adequados de higiene oral e nível de dependência

\begin{tabular}{|c|c|c|}
\hline $\begin{array}{l}\text { Paciente } \\
\text { independente }\end{array}$ & Paciente que pode deambular & $\begin{array}{l}\text { Deslocar-se até uma pia e realizar a própria higiene } \\
\text { Estimular e orientar quanto às técnicas corretas }\end{array}$ \\
\hline Paciente & Pacientes que não podem se deslocar & Oferecer uma cuba para higiene no leito \\
\hline $\begin{array}{l}\text { Parcialmente } \\
\text { dependente }\end{array}$ & Pacientes com dificuldades motoras & $\begin{array}{l}\text { Recursos auxiliares como escovas com cabo adaptado, escovas elé- } \\
\text { tricas... }\end{array}$ \\
\hline Paciente dependente & Pacientes com impossibilidades motoras & $\begin{array}{l}\text { Higiene realizada por um cuidador ou pela enfermagem com esco- } \\
\text { vas comuns ou escovas elétricas }\end{array}$ \\
\hline & Paciente intubado & Escovação e higiene com gaze e anti-séptico do tipo clorexidina $0,12 \%$ \\
\hline
\end{tabular}


gienização bucal. O mesmo procedimento era realizado para todos os pacientes. Raramente, escovas dentais eram usadas pelos próprios indivíduos, os quais se encontravam totalmente independentes de cuidados especiais, sem orientação ou incentivo de membros da equipe que os assistia. ${ }^{(19-21)}$

Com base em nossa pesquisa foi possível perceber que a equipe de enfermagem responsável pelos cuidados de higiene dos pacientes hospitalizados recebeu poucas informaçóes a respeito dos métodos de controle de placa responsáveis pela origem das principais patologias bucais e que, ainda, desconhece vários recursos de higiene bucal que poderiam ser utilizados no ambiente hospitalar com significativas melhorias na manutenção e recuperação da saúde bucal desses indivíduos. ${ }^{(14-16)}$ Quanto à freqüência com que sáo realizados os cuidados específicos de higiene bucal, são variadas as opiniōes encontradas por diferentes autores pesquisados. ${ }^{(1,3,14)} \mathrm{A}$ escovação utilizando escova e pasta dental deve ser preconizada como cuidado básico essencial, para que possa ser estendida a todos os pacientes que precisam receber esse tipo de atenção. Observamos que procedimentos de higiene bucal eram realizados apenas duas vezes ao dia, nas UTIs escolhidas para nosso estudo, coincidindo com o horário do banho pouco antes de iniciar o intervalo de tempo destinado às visitas da família e acompanhantes.

Alguns autores afirmam que a melhor prevenção é a remoção mecânica da placa pela escovação, pelo uso do fio dental e por outros meios de higiene. São descritas na literatura diversas técnicas de escovação dentária e, se compararmos o efeito da limpeza dentária, nenhum método mostra-se superior a outro, como descreve. ${ }^{(4,5,7)}$ Estes autores afirmam que a disposição e a capacidade do paciente, bem como os resultados obtidos, independentemente da técnica de escovação utilizada, são fatores mais importantes do que a seleção do método de escovação dentária. Em 100\% das UTIs visitadas a única técnica de higienização bucal utilizada por equipes de enfermagem era com o uso de bastóes envoltos por gaze embebida em cloreto de cetilpiridínio. Nossa pesquisa reconheceu que, de fato a higienização com escova para pacientes total ou parcialmente dependentes exige maiores habilidades e consome maior espaço de tempo. Esta parece ser uma das razóes para a aplicação dos cuidados que vêm acontecendo nos hospitais visitados. Recomenda-se que os cuidados efetuados em UTIs sejam revistos, em benefício dos pacientes.

\section{CONCLUSÃO}

Com base nos resultados obtidos, chegou-se a algumas conclusóes:

Para manter a saúde oral em níveis satisfatórios, são necessárias modificaçóes nos cuidados dispensados, atualmente, no ambiente hospitalar pelas equipes de enfermagem;
As equipes de enfermagem estudadas pouco conhecem a respeito dos métodos de controle de placa responsável pelas principais patologias bucais e dos diversos produtos que podem ser utilizados na higiene bucal;

Os recursos disponíveis nos hospitais visitados são escassos e impróprios, impossibilitando a manutenção da saúde oral e dificultando o controle de infecçôes bucais já instaladas;

De acordo com as respostas recebidas, os profissionais entrevistados não receberam formação adequada para realizar procedimentos de cuidados bucais. Dessa forma, sugerimos que os currículos de enfermagem sejam revistos e que os conhecimentos em odontologia preventiva sejam mais bem difundidos nessa classe de profissionais;

A presença de um cirurgião-dentista é sugerida como uma tentativa de solucionar as dificuldades apresentadas na manutenção da saúde oral e no tratamento de doenças bucais que afetam a saúde geral dos indivíduos hospitalizados em UTIs;

A interdisciplinaridade, no atendimento em UTIs, deveria contemplar a presença de cirurgióes-dentistas integrados aos princípios das equipes envolvidas. Os conhecimentos difundidos no ambiente hospitalar seriam proveitosos, inclusive após a alta hospitalar, visando à obtenção da qualidade de vida para estes pacientes.

\section{ABSTRACT}

Objectives: A study was carried out, by means of a questionnaire for guided interviews seeking to establish a profile of perceptions and oral care given by ICU nursing team to patients in intensive care units.

Methods: The target population consisted of nursing practitioners divided in three education categories: nurses, nursing technicians and auxiliary nurses working at public and private hospital institutions providing for intensive care unit patients in Belem-PA. Dentistry experience has developed in this field reporting scientific findings and practical applications on prevention and reestablishment of the oral health in question.

Results: This survey disclosed results suggesting that oral hygiene care given to intensive care units patients is insufficient and inadequate requiring changes be made in the care now provided in the nosocomial environment by the nursing team.

Conclusion: Presence of a dentist, knowledge of preventive dentistry, dissemination and use of oral hygiene specific resources are means suggested in an attempt to resolve difficulties found in oral health maintenance and treatment of oral diseases that affect the general health of hospitalized individuals. Interdisciplinary action for these individuals is advocated to achieve a better quality of life by preventing or minimizing oral pathologies.

Keywords: Patient care team; Oral hygiene/nursing; Intensive care units 


\section{REFERÊNCIAS}

1. Abidia RF. Oral care in the intensive care unit: a review. J Contemp Dent Pract. 2007;8(1):76-82.

2. Morais TMN, Silva A, Avi ALRO, Souza PHR, Knobel E, Camargo LFA. A importância da atuação odontológica em pacientes internados em unidade de terapia intensiva: [revisão]. Rev Bras Ter Intensiva. 2006;18(4):412-7.

3. Jenkins D. Oral care in the ICU: an important nursing role. Nurs Stand. 1989;4(7):24-8.

4. Lindhe J. Tratado de periodontia clínica e implantodontia oral. 3a. ed. Rio de Janeiro: Guanabara Koogan; 1999.

5. Cardeñosa Cendrero JA, Solé-Violán J, Bordes Benítez A, Noguera Catalán J, Arroyo Fernández J, Saavedra Santana P, Rodríguez de Castro F. Role of different routes of tracheal colonization in the development of pneumonia in patients receiving mechanical ventilation. Chest. 1999;116(2):462-70.

6. Komiyama K, Tynan JJ, Habbick BF, Duncan DE, Liepert DJ. Pseudomonas aeruginosa in the oral cavity and sputum of patients with cystic fibrosis. Oral Surg Oral Med Oral Pathol. 1985;59(6):590-4.

7. Scannapieco FA, Papandonatos GD, Dunford RG. Associations between oral conditions and respiratory disease in a national sample survey population. Ann Periodontol. 1998;3(1):251-6.

8. Treolar DM, Stechmiller JK. Use of a clinical assessment tool for orally intubated patients. Am J Crit Care. 1995;4(5):355-60.

9. Liwu A. Oral hygiene in intubated patients. Aust J Adv Nurs. 1990;7(2):4-7.

10. McNeill HE. Biting back at poor oral hygiene. Intensive
Crit Care Nurs. 2000;16(6):367-72.

11. Hayes J, Jones C. A collaborative approach to oral care during critical illness. Dent Health (London). 1995;34(3):6-10.

12. Potter PA, Perry AG. Fundamentos de enfermagem: conceitos, processo e prática. 4a. ed. Rio de Janeiro: Guanabara Koogan; 1999.

13. Kite K, Pearson L. A rationale for mouth care: the integration of theory with practice. Intensive Crit Care Nurs. 1995;11(2):71-6.

14. Berry AM, Davidson PM. Beyond comfort: oral hygiene as a critical nursing activity in the intensive care unit. Intensive Crit Care Nurs. 2006;22(6):318-28.

15. Logan HL, Ettinger R, McLeran H, Casko R, Dal Secco D. Common misconceptions about oral health in the older adult: nursing practices. Spec Care Dentist. 1991;11(6):243-7.

16. Blank LW, Arvidson-Bufano UB, Yellowitz JA. The effect of nurses' background on performance of nursing home resident oral health assessments pre-and post-training. Spec Care Dentist. 1996;16(2):65-70.

17. Puricelli E, Wells $\mathrm{H}$. A face hospitalar da odontologia. Rev ABO Nac. 1996; 4(1):8-13.

18. Atkinson LD, Murray ME. Fundamentos de enfermagem: introdução ao processo de enfermagem. Rio de Janeiro: Guanabara Koogan; c1989.

19. Erickson L. Oral health promotion and prevention for older adults. Dent Clin North Am. 1997;41(4):727-50.

20. Binkley C, Furr LA, Carrico R, McCurren C. Survey of oral care practices in US intensive care units. Am J Infect Control. 2004;32(3):161-9.

21. Wall RJ, Dittus RS, Ely EW. Protocol-driven care in the intensive care unit: a tool for quality. Critcal Care. 2001;5(6):2835. Comment on: Crit Care. 2001;5(6):349-54. 